\title{
Characterization of kinesin-like proteins in silkworm posterior silkgland cells
}

Qiao Wang ${ }^{1}$, Junlin Teng ${ }^{1}$, Birong Shen ${ }^{1}$, Wei Zhang ${ }^{1}$, Yige Guo ${ }^{1}$, Xiaolei Su ${ }^{1}$, Chuanxi Zhang ${ }^{3}$, Albert CH Yu ${ }^{4}$, Jianguo Chen ${ }^{1,2}$

${ }^{I}$ The Key Laboratory of Cell Proliferation and Differentiation of Ministry of Education, The State Key Laboratory of Bio-membrane and Membrane Bio-engineering, College of Life Sciences, Peking University, Beijing 100871, China; ${ }^{2}$ The Center for Theoretical Biology, Peking University, Beijing 100871, China; ${ }^{3}$ Institute of Insect Sciences, Zhejiang University, Hangzhou 310029, China; ${ }^{4}$ Department of Neurobiology, Neuroscience Research Institute, School of Basic Medical Sciences, The Key Laboratory for Neuroscience (Ministry of Education and Ministry of Public Health), Peking University, Beijing 100191, China

Kinesins are microtubule-based motors involved in various intracellular transports. Neurons, flagellated cells, and pigment cells have been traditionally used as model systems to study the cellular functions of kinesins. Here, we report silkworm posterior silkgland (PSG), specialized cells with an extensive endomembrane system for intracellular transport and efficient secretion of fibroin, as a novel model for kinesin study. To investigate kinesindriven intracellular transport in PSG cells, we cloned five silkworm kinesin-like proteins (KLPs), BmKinesin-1, BmKinesin-6, BmKinesin-7, BmKinesin-13, and BmKinesin-14A. We determined their expression patterns by relative real-time PCR and western blotting. Immunofluorescence microscopy verified their colocalization with microtubules. By combining pull-down assays, LC-MS/MS, and western blotting analysis, we identified many potential cargoes of BmKinesin-1 in PSG, including fibroin-containing granules and exuperantia-associated ribonucleoprotein (RNP) complexes. Moreover, BmKinesin-13 overexpression disrupted the microtubule network in BmN cells, which is consistent with a role of Kinesin-13 in regulating microtubule dynamics in other organisms. On the basis of these results, we concluded that PSG might have advantages in elucidating mechanisms of intracellular transport in secretory tissues and could serve as a potential model for kinesin studies.

Keywords: Kinesin-like proteins, posterior silkgland cells, intracellular transport, exuperantia, fibroin granules, BmKinesin-1 Cell Research (2010) 20:713-727. doi:10.1038/cr.2010.47; published online 6 April 2010

\section{Introduction}

The first kinesin, isolated from squid giant axon, was

Correspondence: Junlin Teng ${ }^{\mathrm{a}}$, Jianguo Chen ${ }^{\mathrm{b}}$

Tel: +86-10-62755786; Fax: +86-10-62755786

a'E-mail: junlinteng@pku.edu.cn

bE-mail: chenjg@pku.edu.cn

Abbreviation: Bm (Bombyx mori); BmNPV (Bombyx mori nucleopolyhedrovirus); CBD (cargo-binding domain); Dm (Drosophila melanoganster); ER (endoplasmic reticulum); EGFP (enhanced green fluorescent protein); GST (glutathione S-transferase); KHC (kinesin heavy chain); KLC (kinesin light chain); KLP (kinesin-like protein); MSG (middle silkgland); PCD (programmed cell death); PSG (posterior silkgland); RNP (ribonucleoprotein); RpL3 (ribosomal protein L3); UTR (untranslated region); EYFP (enhanced yellow fluorescent protein).

Received 5 November 2009; revised 24 December 2009; accepted 28 January 2010; published online 6 April 2010 discovered to be responsible for fast axonal transport [1]. As more and more kinesins were cloned, phylogenetic analysis has been performed and 14 kinesin subfamilies have now been identified across phyla [2, 3]. Among them, the conventional kinesin (kinesin heavy chain, $\mathrm{KHC}$ ), a homodimer in Kinesin-1 subfamily, has been investigated extensively. It contains an $\mathrm{N}$-terminal motor domain, an internal coiled-coil region, and a C-terminal cargo-binding domain (CBD). It moves along microtubules in a 'hand-over-hand' mechanism [4], with each step gated by adenosine triphosphate hydrolysis [5]. Kinesin-2 transports protein complexes [6], Kinesin-13 regulates microtubule dynamics by serving as a microtubule depolymerase [7-9], while Kinesin-14 organizes microtubules into parallel bundles and facilitates spindle assembly in mitosis [10-13]. Substantial evidence has 
indicated that the kinesin superfamily proteins have significant roles in intracellular transport, cell division, and critical developmental processes [14].

Neurons, flagellated cells, and pigment cells have been traditionally used as model systems to study the kinesindriven intracellular transport. Neurons are one of the most important model systems, in which various kinesindependent axonal or dendritic transport pathways have been intensively investigated [14, 15]. Flagellar kinesins empower the anterograde movement of the intraflagellar transport particles for the assembly and maintenance of eukaryotic cilia and flagella [16]. Studies of kinesins in pigment cells revealed the mechanism of the intracellular transport of pigment granules [17]. However, many more intracellular transport mechanisms remain elusive. One of the major obstacles lies in the relatively low abundance of kinesins and their associated factors in the currently available models, making it difficult to identify novel protein factors involved in these intracellular transports. Therefore, it requires the establishment of new models, in which protein interaction network for regulating intracellular transport could be identified.

Silkworm, Bombyx mori $(\mathrm{Bm})$, produces silk, with its posterior silkgland (PSG) cells devoting $85 \%$ of their protein synthesis activities to silk production [18]. These highly specialized and polarized PSG cells secrete fibroin, one of the major silk components, into the PSG lumen $[19,20]$. Fibroin is composed of heavy chain (molecular weight $350 \mathrm{kDa}$ ), light chain (molecular weight $26 \mathrm{kDa}$ ), and P25 with a molar ratio of 6:6:1 [20].

Silkworm PSG may have several advantages for studying the kinesin-driven intracellular transport. Silkworms are suitable for large-scale culture. Their large-sized PSGs [19] are relatively easy to dissect for biochemical analysis, such as protein purification and pull-down analysis. Silkworm genomic sequences and cDNA databases are available [21, 22]; hence, the cDNA cloning of kinesins and their associated proteins is no longer difficult. Most importantly, PSG is a highly differentiated secretory tissue, with a complex and extensive cytoskeleton system and endomembrane system (Golgi apparatus, ER, and secretory granules) occupying a large portion of the cytoplasm [19, 23]. These PSG cells are responsible for the active intracellular transport during secretion [24], which may require a broad participation of kinesins and other transport-specific proteins. In addition, RNAi technology recently established in silkworm [25] has enabled researchers to knockdown target gene transcripts by injection of dsRNA into adult or juvenile insects. Thus, RNAi technology, in combination with the BmNPV baculoviruses transfection system [26], transgenic silkworm technique [27], and micromanipulation tools in silkworm cells [28], offers a great opportunity to assess the physiological function of individual protein in certain cellular processes and developmental stages. Consequently, we think that PSG will supplement the well-established studies of fruit fly and provide researchers with great opportunities for identifying novel secretory proteins and pathways.

In this study, we investigated the possibility of establishing silkworm PSG as a novel model to study the kinesin-driven intracellular transport. However, only one partial sequence of silkworm kinesin-like protein (KLP) has been previously reported [29], compared to 45 kinesin superfamily proteins in humans, rats, and mice [3], and 24 in Drosophila melanogaster (Dm) [2]. Therefore, we isolated and characterized five silkworm KLPs (BmKinesin-1, BmKinesin-6, BmKinesin-7, BmKinesin-13, and BmKinesin-14A), and further provided evidence suggesting that BmKinesin-1 might transport fibroincontaining vesicles and exuperantia-associated ribonucleoprotein (RNP) complexes.

\section{Results}

\section{Identification of KLPs in silkworm silkgland}

The motor domains of all kinesins identified so far contain a homologous region of about 350 amino acids. Within this area, several highly conserved motifs, such as IFAYGQT and LVDLAGSE, are present across species and are considered as sequence markers of kinesins [30]. Here, we scanned the silkworm genome [21] and EST database [22] to look for these sequence markers, and successfully located five homologous sequences (Supplementary information, Figure S1A). We extended the conserved sequences forward and backward (Supplementary information, Figure S1B), either by applying Basic Local Alignment Search Tool (BLAST) in silkworm cDNA and genome database or by comparing sequences between $\mathrm{Dm}$ and $\mathrm{Bm}$. We succeeded in predicting the start codon, the stop codon, the $5^{\prime}$-untranslated region (UTR) and the 3'-UTR, and finally retrieved the entire nucleotide sequences of five KLPs. These predictions were further verified by reverse transcription PCR (RT-PCR) amplification from PSG cDNA. According to the standardized kinesin nomenclature [2], we named these five KLPs, BmKinesin-1, BmKinesin-6, BmKinesin-7, BmKinesin-13, and BmKinesin-14A (GenBank accession nos: EF092447-EF092451). Furthermore, we mapped their exon-intron structures (Supplementary information, Figure S2).

\section{Secondary structure and phylogenic analysis}

To understand the functional domains of these KLPs, 
we analyzed the amino acid sequences and predicted the location of coiled-coil regions and motor domains by SMART and COILS (Figure 1A-1E). The motor domains lie between residues 10 and 341, 55 and 455, 2 and 333, 176 and 517, and 277 and 614 for BmKinesin-1 (Figure 1A), BmKinesin-6 (Figure 1B), BmKinesin-7 (Figure 1C), BmKinesin-13 (Figure 1D), and BmKinesin-14A (Figure 1E), respectively.

To compare the functional similarities of these five silkworm KLPs with those already characterized kinesin members, we constructed a phylogenic tree based on the alignable motor domains [2, 3]. The tree sorted five silkworm KLPs, BmKinesin-1, BmKinesin-6, BmKinesin-7, BmKinesin-13, and BmKinesin-14A to Kinesin-1, Kinesin-6, Kinesin-7, Kinesin-13, and Kinesin-14A subfamilies, respectively (Figure 2).

All these five KLPs cloned from silkworm can be matched with their own orthologues in both Dm and human, sharing high similarity in their motor domains and even their full-length sequences (Supplementary information, Figure S3A and S3B). An identity of $94 \%$ was observed between the motor domains of BmKinesin-1 and DmKHC (GenBank accession no: M24441), and $73 \%$ between their complete sequences. The homologues of BmKinesin-6, BmKinesin-7, BmKinesin-13, and BmKinesin-14A in Dm are DmKLP54E (GenBank accession no: AE003802), DmCmeta (GenBank accession nos: AF220353), DmKLP10A (GenBank accession no: AAN09282), and DmNcd (GenBank accession no: X52814), respectively. The identities of their motor domains and complete sequences are $33 \%$ and $24 \%$ between BmKinesin-6 and DmKLP54E, 50\% and 9\% between BmKinesin-7 and DmCmeta, $81 \%$ and 54\% between BmKinesin-13 and DmKLP10A, 52\% and 32\% between BmKinesin-14A and DmNcd, respectively. The sequence conservation between Bm and Dm kinesins
A

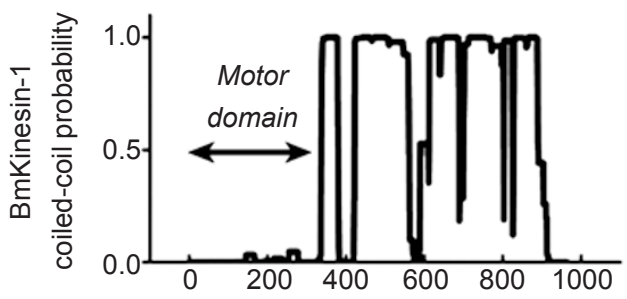

C

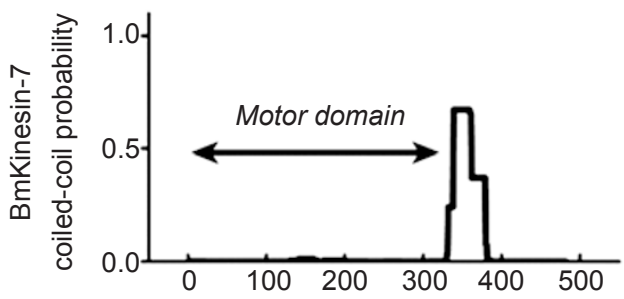

B

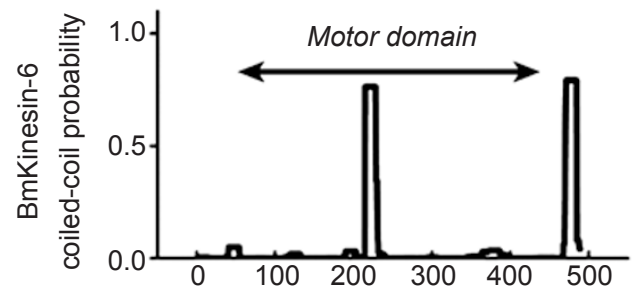

$\mathrm{D}$

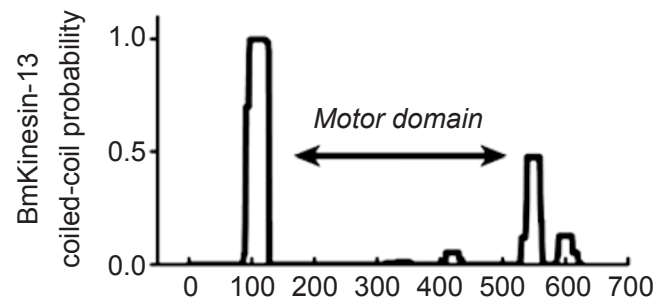

E

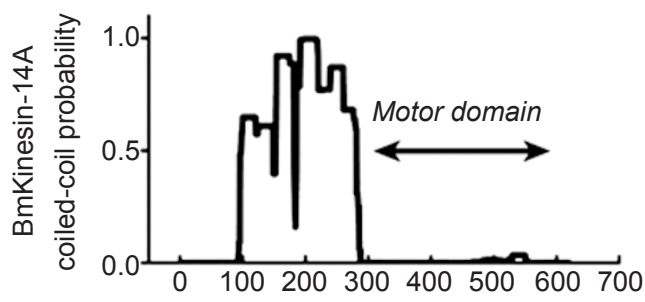

Figure 1 Domain architecture of five silkworm KLPs. (A-E) The motor domains and coiled-coil regions of five silkworm kinesins are indicated in the schematic pictures, which are the combination of the results from SMART and COILS. 


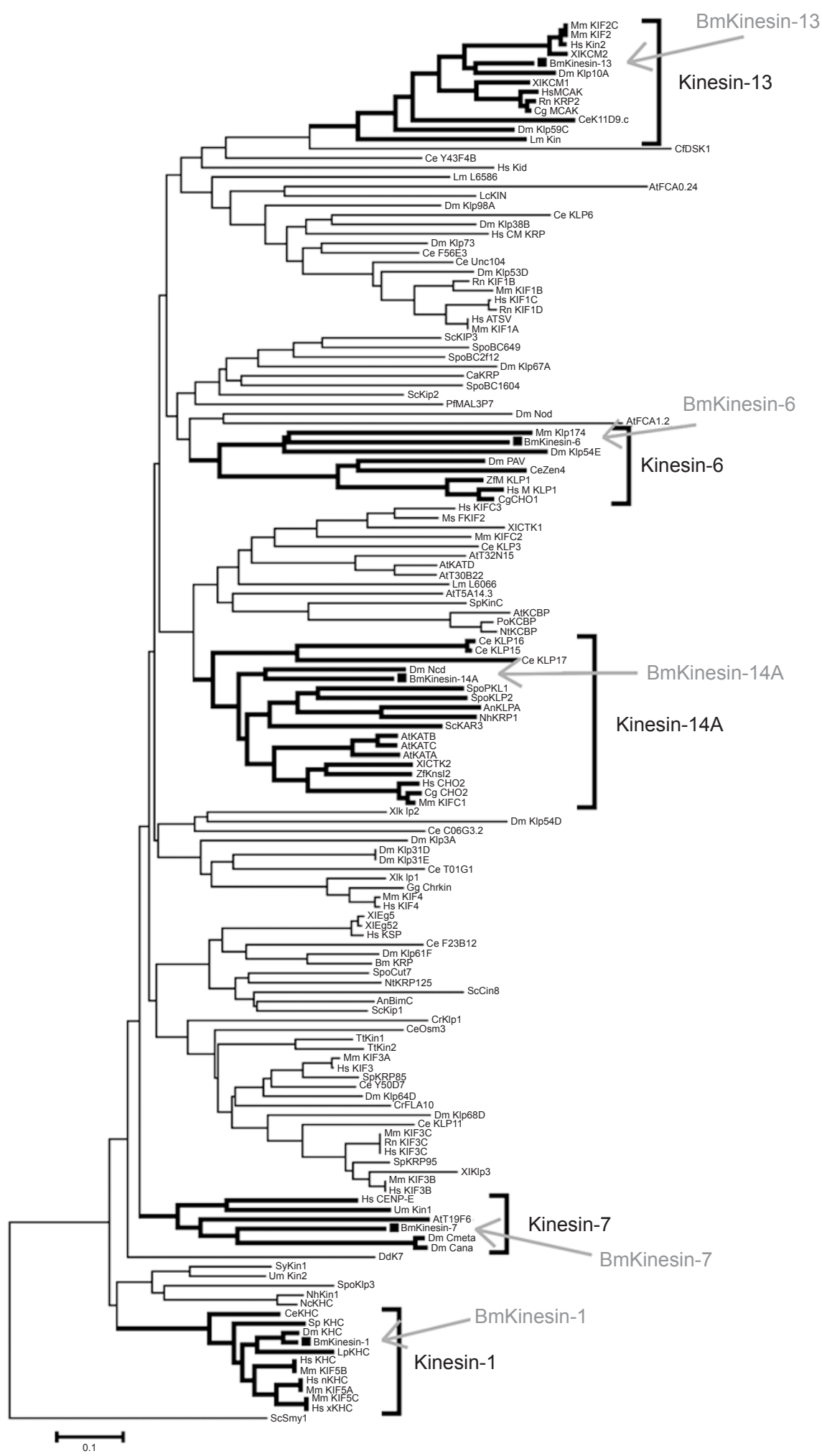

Figure 2 Molecular phylogenetic tree of kinesin superfamily proteins. The five labelled and bracketed subfamilies contain the five silkworm KLPs (arrows). An, Aspergillus nidulans (Emericella nidulans); At, Arabidopsis thaliana; Bm, Bombyx mori; Ce, Caenorhabditis elegans; Cf, Cylindrotheca fusiformis (diatom); Dm, Drosophila melanogaster, Hs, Homo sapiens; Lm, Leishmania major, Mm, Mus musculus; Pf, Plasmodium falciparum; Sc, Saccharomyces cerevisiae; and Spo, Schizosaccharomyces pombe. 
A
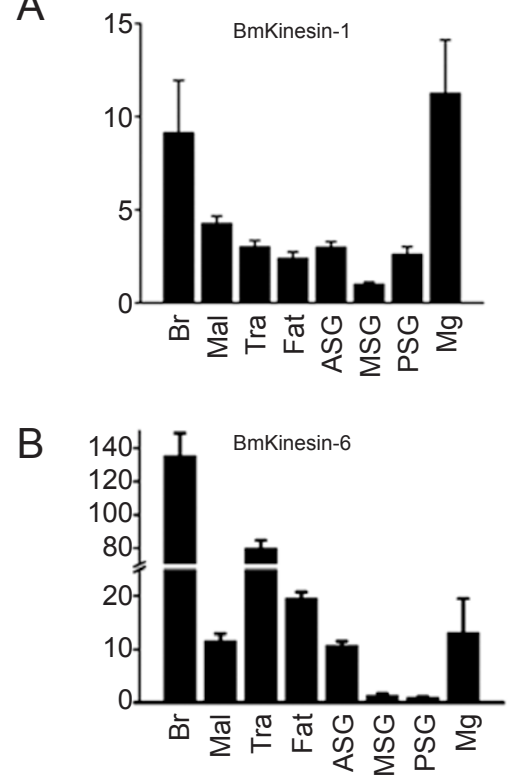

C

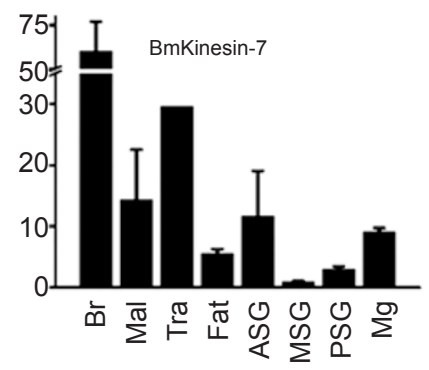

D

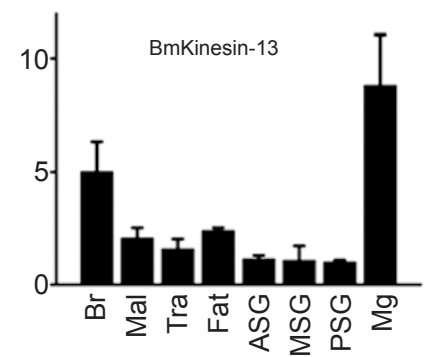

E

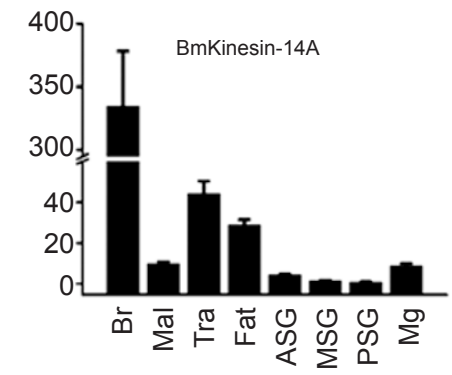

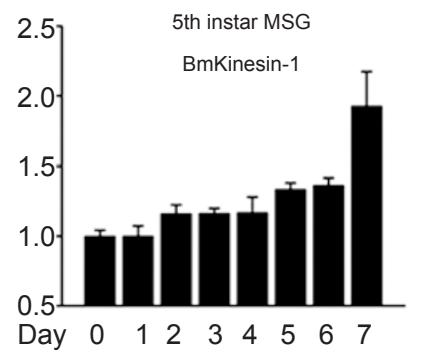
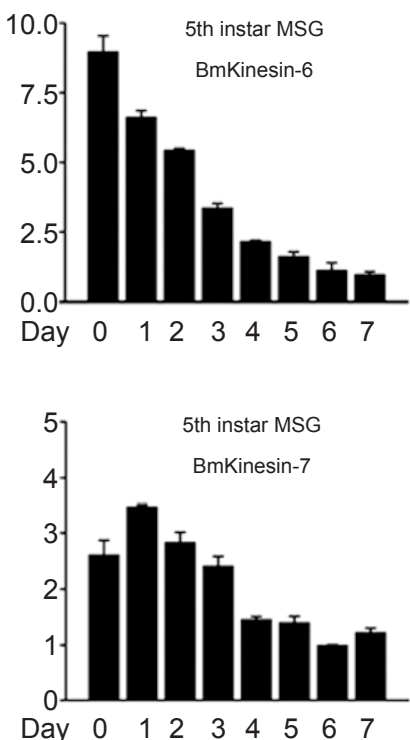

Day $0 \begin{array}{llllllll} & 1 & 2 & 3 & 4 & 5 & 6 & 7\end{array}$
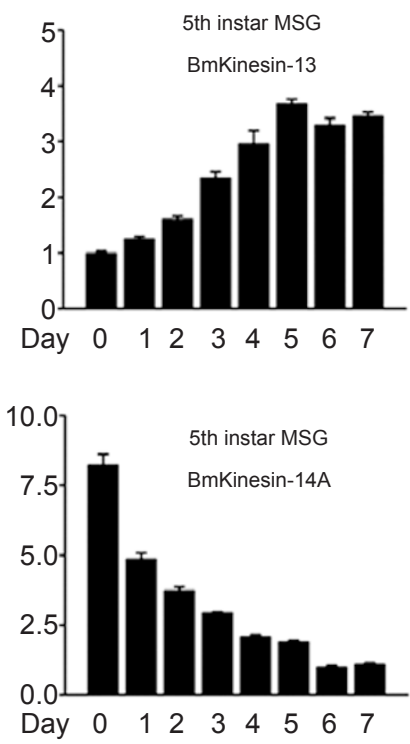
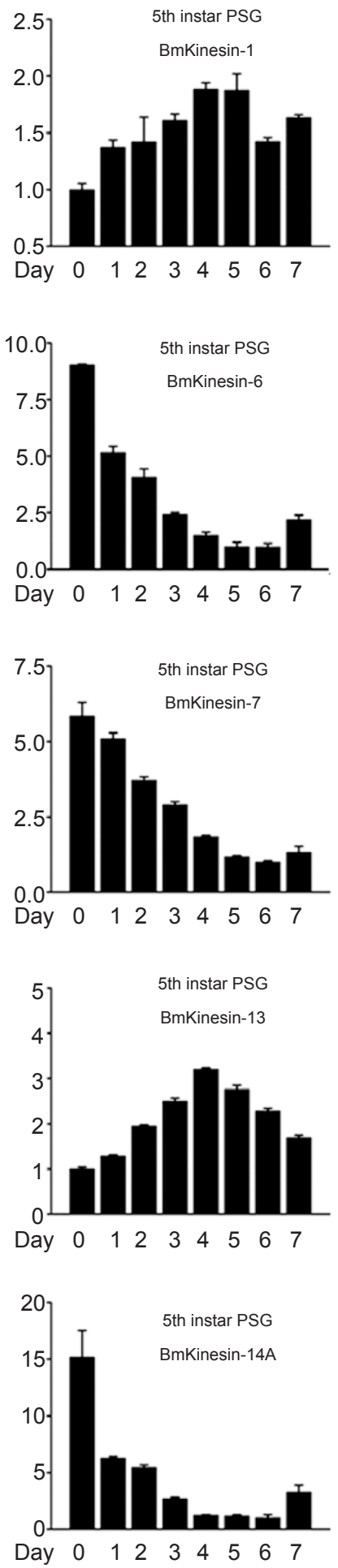

Figure 3 Relative mRNA expression levels of five silkworm KLPs. (A-E) Relative mRNA expression pattern of BmKinesin-1 (A), BmKinesin-6 (B), BmKinesin-7 (C), BmKinesin-13 (D), and BmKinesin-14A (E). Every row contains three bar graphs. Left: relative mRNA expression levels of five cloned KLPs in eight different tissues of silkworm, brain (Br), Malpighian tubule (Mal), trachea (Tra), fat body (Fat), anterior silkgland (ASG), middle silkgland (MSG), posterior silkgland (PSG), and midgut (Mg). Middle: real-time PCR analysis in different developmental stages of MSG. Right: mRNA expression levels in PSG development. Days 0-7 indicated as day 0-7 of the fifth-instar larvae. 
suggests their conserved physiological functions in kinesin-driven intracellular transport. Therefore, silkworm could be used to supplement studies in fruit fly.

Tissue distribution and developmental expression patterns in silkgland

To determine the tissue distribution of these five KLPs, we measured their mRNA levels by relative realtime PCR in eight different tissues from the fifth-instar day-3 larvae. All the KLPs were expressed ubiquitously, but with higher expression levels in the brain (Figure 3A-3E, left); BmKinesin-14A transcripts were dominantly expressed in the silkworm brain, and were ubiquitously detected throughout the whole brain on the fifthinstar day-3 brains by whole-mount in situ hybridization (Supplementary information, Figure S4). The transcripts of BmKinesin-13 and BmKinesin-1 were enriched in the midgut (Figure 3A and 3D, left), while the relative expression level of BmKinesin-6 was higher in the trachea (Figure 3B, left). In the fifth-instar middle silkgland (MSG) and PSG, the mRNA levels of these five KLPs displayed different patterns of temporal variation. The transcripts of BmKinesin-6, BmKinesin-7, and BmKinesin-14A exhibited a similar trend of decline in both the fifth-instar MSG and PSG (Figure 3B, 3C and 3E middle/right), whereas BmKinesin-1 and BmKinesin-13 mRNAs tended to be gradually accumulated (Figure 3A and $3 \mathrm{D}$, middle/right).

To confirm the real-time PCR results at the protein level, we produced rabbit and mouse polyclonal antibodies against BmKinesin-1 (Supplementary information, Figure S5). Western blotting analysis of BmKinesin-1 demonstrated that the change observed in protein levels (Figure 4) correlated well with that at mRNA levels. Most importantly, both mRNA and protein levels of BmKinesin-1 were stably increased in PSG of the fifthinstar larvae from day 0 to day 5 (Figures $3 \mathrm{~A}$ and $4 \mathrm{C}$ ). However, during the last 2 days of the fifth-instar in PSG, the relative mRNA levels of both BmKinesin-1 and BmKinesin-13 (Figure 3A and 3D, right), and the protein levels of both BmKinesin-1 and tubulin decreased (Figure $4 \mathrm{C})$.

\section{Subcellular localization}

Next, we used the Bm nucleopolyhedrovirus (BmNPV) baculovirus expression system and immunofluorescence microscopy to investigate the subcellular localization of these five silkworm kinesins. Recombinant BmNPV baculoviruses expressing BmKLP-EGFP fusion proteins were generated. Confocal microscopy revealed various extents of colocalization with microtubules, with the exception of BmKinesin-7, in the infected BmN cells

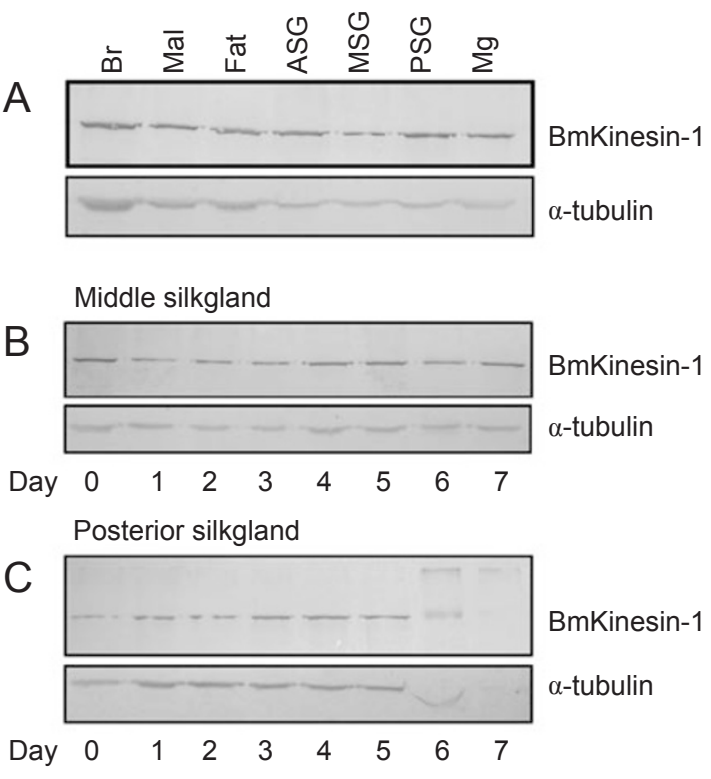

Figure 4 Protein expression pattern of BmKinesin-1. Expression levels of BmKinesin-1 in different tissues (A) and in the fifthinstar developmental MSG/PSG (B and C) were detected by anti-BmKinesin-1 and anti-tubulin antibodies. Brain (Br), Malpighian tubule (Mal), fat body (Fat), anterior silkgland (ASG), middle silkgland (MSG), posterior silkgland (PSG), and midgut $(\mathrm{Mg})$. Days 0-7 indicated as day 0-7 of the fifth-instar larvae.

(Figure 5A-5E). Interestingly, compared with $2 \%$ for BmKinesin- $1,7 \%$ for BmKinesin- 6 , and $0.5 \%$ for BmKinesin-13, the percentage of colocalization of BmKinesin$14 \mathrm{~A}$ and microtubules in the infected $\mathrm{BmN}$ cells was as high as $74 \%$ (Figure $5 \mathrm{~F}$ ), possibly explained by a recent report that Kinesin-14s bind to microtubules through their motor and tail domains to organize microtubules into parallel bundles [12, 13].

We further produced polyclonal antibodies to trace the native subcellular localization of BmKinesin-1 and BmKinesin-13 in BmN cells (Supplementary information, Figures S5 and S6). Both endogenous BmKinesin-1 and BmKinesin-13 were enriched and dispersed throughout the cytoplasm (Supplementary information, Figure S7A and S7B). Confocal microscopy demonstrated that speckles of BmKinesin-1 were scattered throughout the cytoplasm from the peri-nuclear region to the periphery of BmN cells (Supplementary information, Figure S7C). Detailed analysis revealed that some BmKinesin-1 localized with vesicle-like structures (Supplementary information, Figure S7C), which was consistent with that of Kif5B in HeLa cells [31].

We also generated baculoviruses BmNPV-BmKinesin1-EGFP and BmNPV-BmKinesin-14A-EYFP to infect 

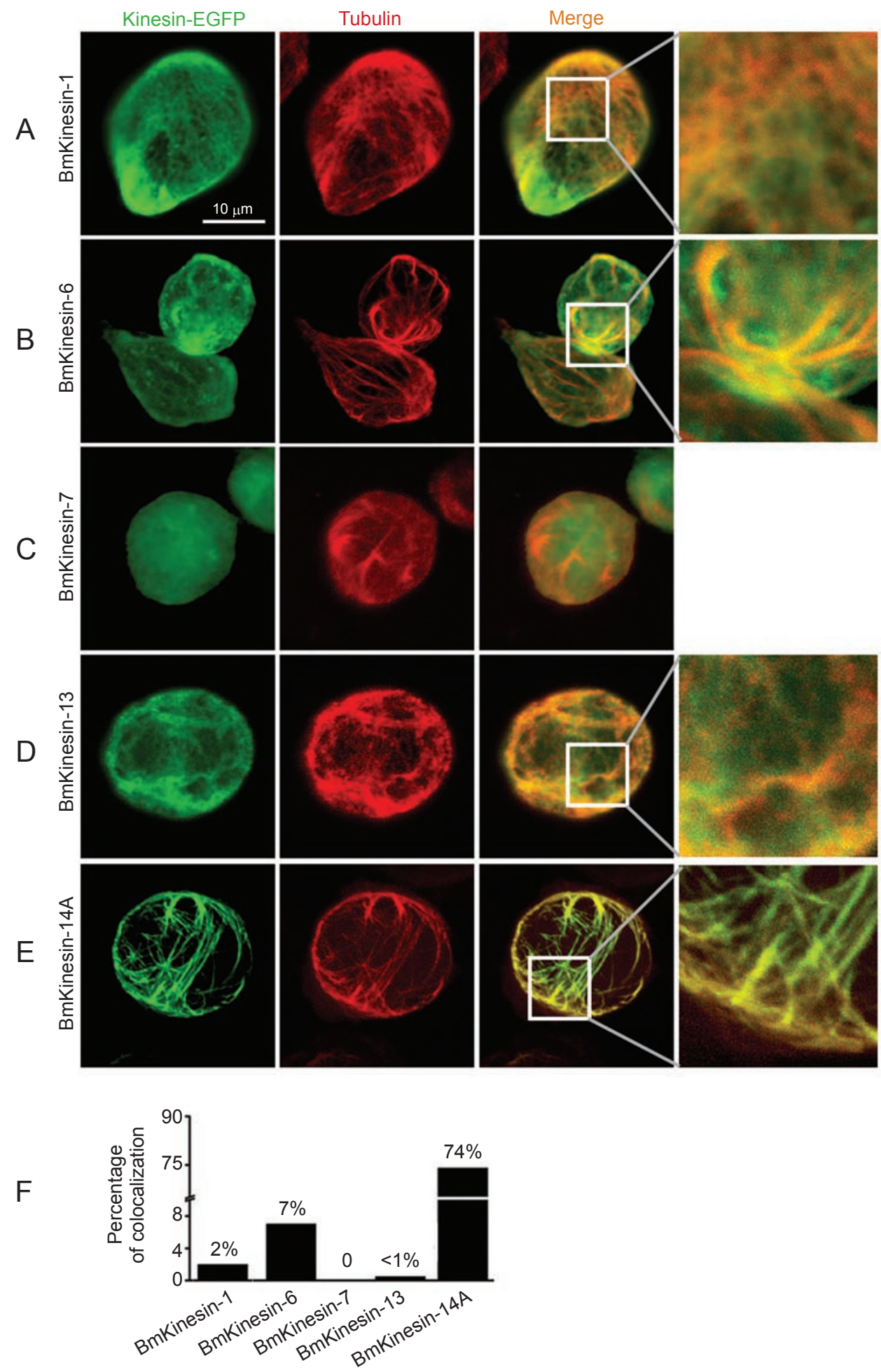

Figure 5 Subcellular distribution of silkworm kinesins in silkworm BmN cells. (A-E) Recombinant baculoviruses-infected BmN cells expressing BmKinesin-1-EGFP (A), BmKinesin-6-EGFP (B), BmKinesin-7-EGFP (C), BmKinesin-13-EGFP (D), and BmKinesin-14A-EGFP (E), with microtubules (red) are immunostained. Colocalization between microtubules and BmKinesin-1, BmKinesin-6, BmKinesin-13, and BmKinesin-14A was observed. The rectangle areas are magnified in the right panel. (F) Statistical graph of colocalization. We calculated the percentage of colocalization. 
silkworm larvae to examine the distribution of BmKinesin-1 and BmKinesin-14A in PSG cells. One week after viral infection, PSGs were dissected and incubated in TC-100 insect medium for confocal microscopy imaging (Figure 6A). BmKinesin-1-EGFP (Figure 6B) and BmKinesin-14A-EYFP (Figure 6C) formed fluorescent radial and circular filamentous networks in infected PSG cells, reminiscent of radial and circular microtubule systems previously reported in PSG cells [23]. The identical and resembling fluorescent filamentous patterns of BmKinesin-1 and BmKinesin-14A to the two microtubule systems further confirmed their affinity with cytoplasmic microtubules.
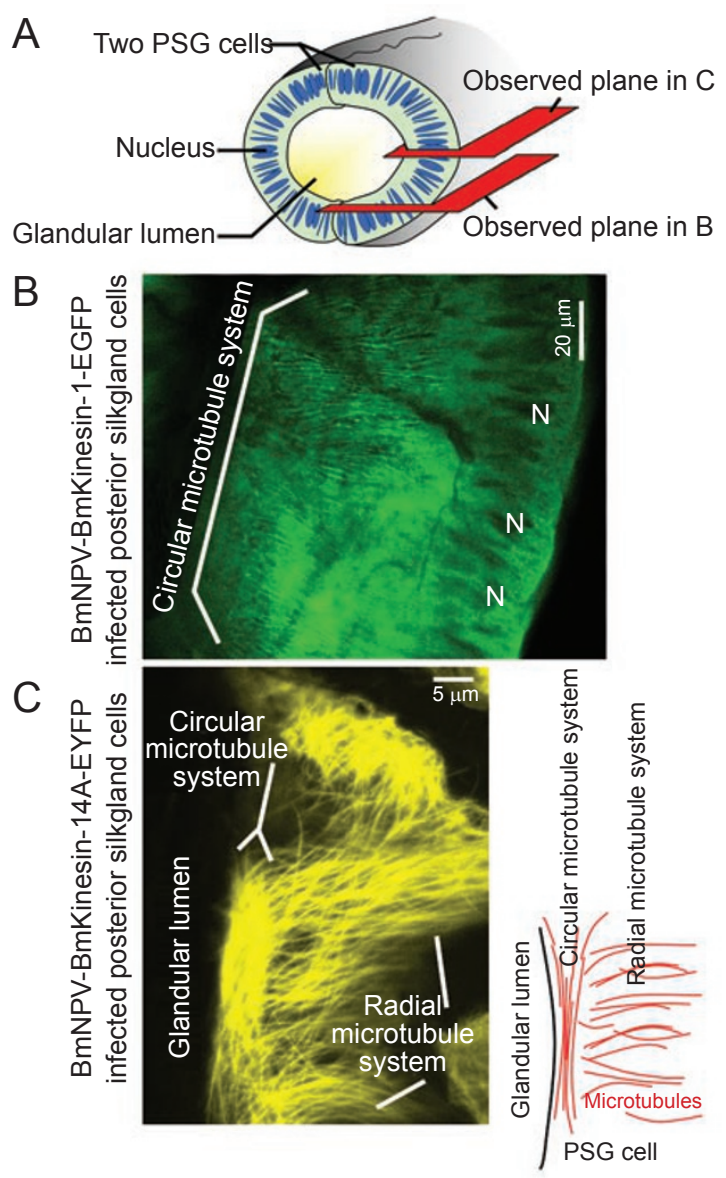

Figure 6 BmKinesin-1 and BmKinesin-14A colocalize with microtubules in PSG cells. (A) Schematic representation of PSG. Two PSG cells surround to form a glandular lumen. (BC) Confocal microscopy of PSG cells infected by BmNPVBmKinesin-1-EGFP (B) and BmNPV-BmKinesin-14A-EYFP (C). Both $\mathbf{B}$ and $\mathbf{C}$ show longitudinal observation plane marked in $\mathbf{A}$. The microtubule network was visualized by the overexpressed BmKinesin-1-EGFP and BmKinesin-14A-EYFP. The boundary between PSG cells and glandular lumen is indicated by the dashed line; $N$ represents nuclei; the schematic picture at right shows two microtubule systems in PSG cells (C).
BmKinesin-1 is associated with fibroin granules and RNP complexes in PSG

The initial characterization of BmKinesin-1 indicated its conserved function as a motor protein for intracellular transport in PSG. Fibroin, synthesized in PSG cells and secreted into the lumen, consists of a heavy chain, a light chain (L-chain) and a glycoprotein P25 [20]. To examine whether BmKinesin-1 interacts with fibroin-containing vesicles in PSG cells, we produced a rabbit polyclonal antibody of fibroin L-chain (FLch) (Supplementary information, Figure S8A and S8B) to perform the pulldown assay. In the glutathione $S$-transferase (GST)-CBD (amino acids 827-964) pull-down experiment (Figure 7A), we probed the pull-down samples with this FLch antibody in western blotting. A specific band of FLch with an approximate molecular weight of $28 \mathrm{kDa}$ was detected in both the GST-BmKinesin-1-CBD pull-down sample and the corresponding PSG crude lysate, but not in the GST pull-down control sample (Figure 7B). Furthermore, we performed indirect immunofluorescence on PSG cryosection and found that BmKinesin-1 partially colocalized with FLch subunit in PSG cells (Figure 7C). These results indicated that BmKinesin-1 interacted with fibroin-containing vesicles.

Moreover, we searched for novel protein-binding partners of BmKinesin-1-CBD in the PSG extracts. Specifically bound proteins were separated by SDS-PAGE and subjected to Coomassie Blue staining, followed by trypsin digestion and LC-MS/MS identification. A series of proteins were identified as potential cargoes of BmKinesin-1 in PSG cells, including RNA-interacting proteins (FKBP45 and exuperantia), nuclear proteins (nucleosome assembly protein and nucleoplasmin), and many ribosomal proteins (ribosomal protein L5, L23A, P0, L3) (Figure 7D and Supplementary information, Figure S9).

\section{Overexpression of BmKinesin-13 disrupts microtubule organization in vivo}

During silkworm development, the enlargement of PSG cells and the enrichment of their intracellular transport systems are thought to satisfy the demands in intensive biosynthesis, transport and secretion [24]. In the meanwhile, the microtubule system is reorganized to fulfil these functional requirements, suggesting the need for a certain remodelling factors. Previous work has revealed that members of Kinesin-13 subfamily are microtubule depolymerases. Kineins-13s form rings [9], rapidly target microtubule ends [32], form a complex at the tips, and depolymerize microtubules processively [7]. Here, since BmKinesin-13 shared a high sequence similarity with its homologues of Kinesin-13s, we speculated that BmKinesin-13 might possess similar activity to 
A

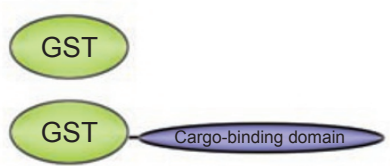

B

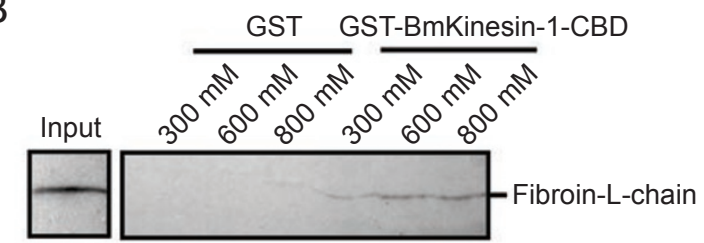

C
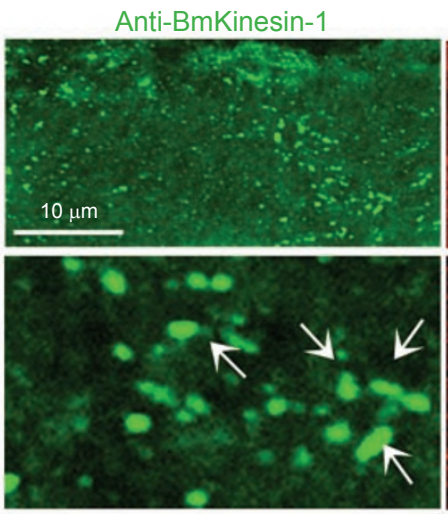

$\mathrm{D}$
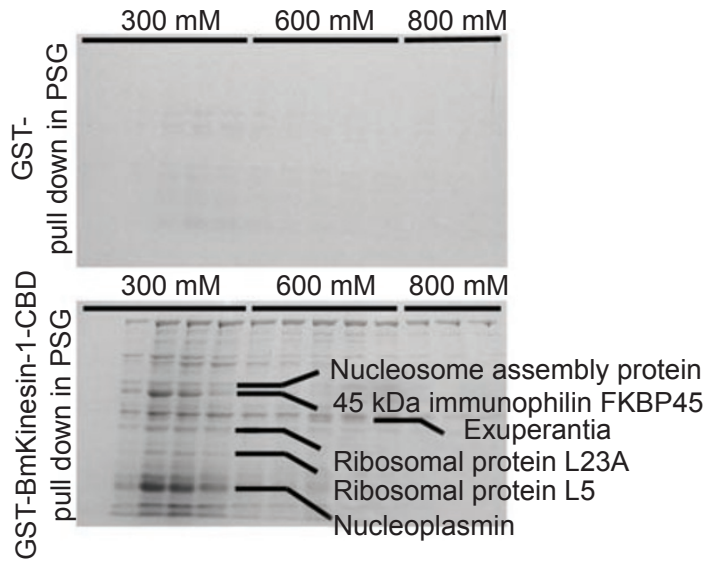

Anti-Fibroin-L-chain
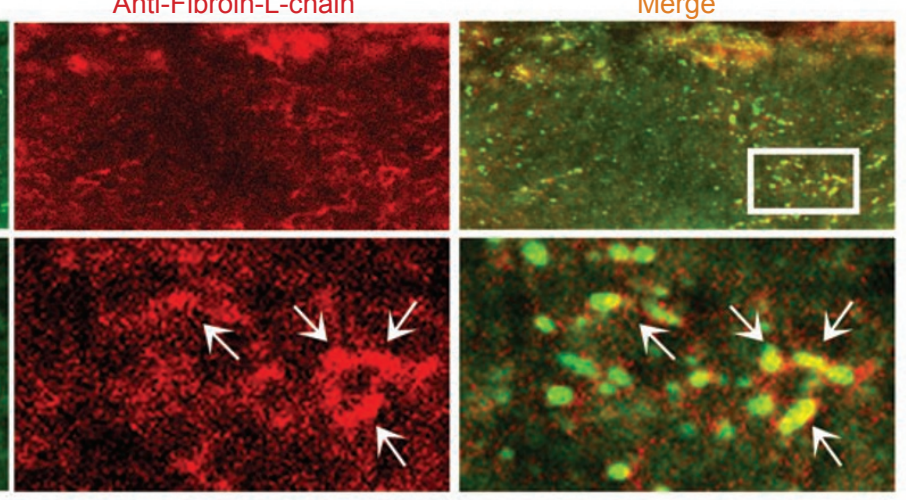

Figure 7 BmKinesin-1-CBD and its interacting molecules. (A) Schematic picture of GST and GST-BmKinesin-1-CBD fusion protein. (B) Interaction of BmKinesin-1 with fibroin-containing granules. The pull-down samples were subjected to western blotting to be probed by anti-fibroin-L-chain antibody. (C) Immunofluorescence of PSG cryosections. PSG cryosections were stained with anti-fibroin-L-chain rabbit polyclonal antibody (red) and anti-BmKinesin-1 mouse polyclonal antibody (green). (D) SDS-PAGE of sequentially eluted samples in the pull-down assay. By stepwise increases of $\mathrm{NaCl}$ concentration, different fractions were eluted in GST- and GST-BmKinesin-1-CBD pull-down columns. Proteins identified by LC-MS/MS are indicated, and exuperantia is the last to be eluted.

disrupt microtubule network. To test this idea, we overexpressed BmKinesin-13 in BmN cells and examined the integrity of the microtubule network. We found that cells transfected with BmNPV-BmKinesin-13-EGFP did not have filamentous microtubules, whereas control cells transfected with only EGFP had an intact microtubule network (Figure 8A-8E). These data support the notion that BmKinesin-13 has a microtubule-destabilizing activity.

To exclude the potential toxicity of baculovirus infection in $\mathrm{BmN}$ cells, we further transfected HeLa cells with BmKinesin-13-EGFP. HeLa cells expressing BmKinesin-13EGFP (with asterisk), but not untransfected controls, no longer had intact microtubule networks (Figure 8F). Thus we further confirmed that the overexpression of BmKinesin-13, like its human orthologues HsKin2 and HsMCAK, could lead to the diffuse distribution of tubulin [32,
33]. Taken together, overexpression of BmKinesin-13 disrupts microtubule organization in vivo and leads to diffuse distribution of tubulin.

\section{Discussion}

The cloning of five KLPs extended our understanding of the silkworm kinesin superfamily. However, we suspect that the total number of KLPs in silkworm can be higher. During the scanning of the silkworm genome and cDNA databases, we located a few more potential kinesin sequence markers, such as IFAYGQT and LVDLAGSE $[30,34]$, though we did not confirm their complete sequences in this study. Furthermore, silkworm and its 'partner' Drosophila share high similarities both in evolutionary rates [35] and kinesin sequences. More than 20 KLPs have been identified in Dm [36]. Therefore, 


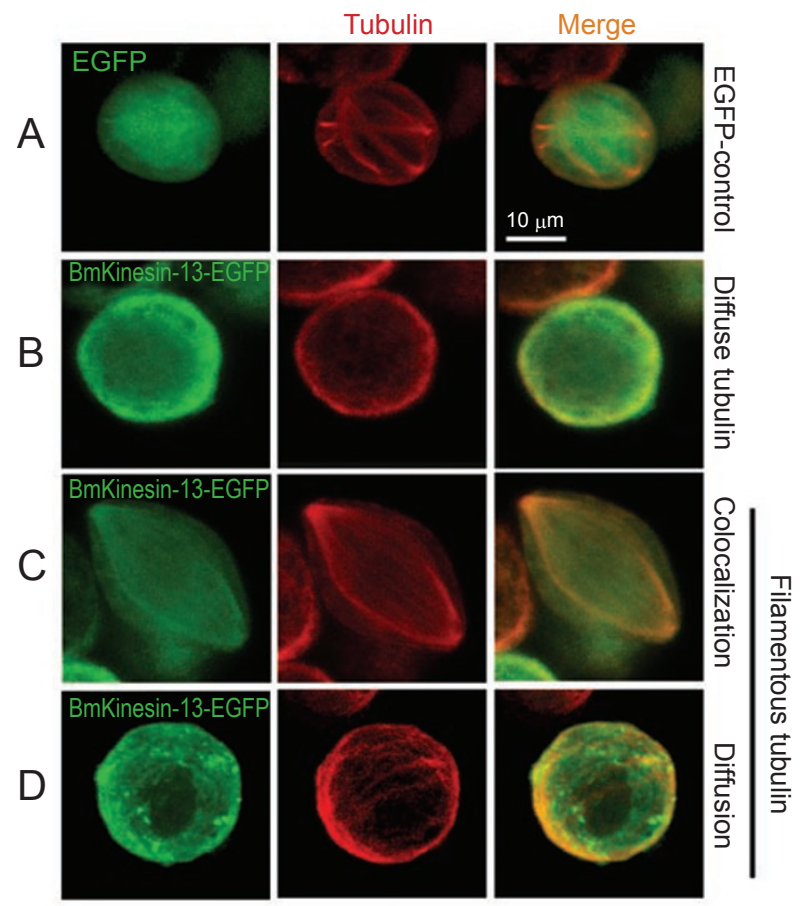

BmKinesin-13-EGFP colocalization with filamentous tubulin BmKinesin-13-EGFP (or EGFP) diffusion with filamentous tubulin $\square$ Diffuse tubulin
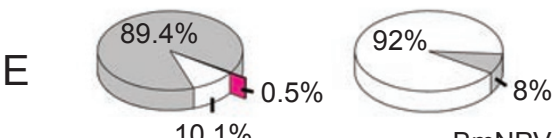

$10.1 \%$ BmNPV-EGFP

BmNPV-BmKinesin-13-EGFP
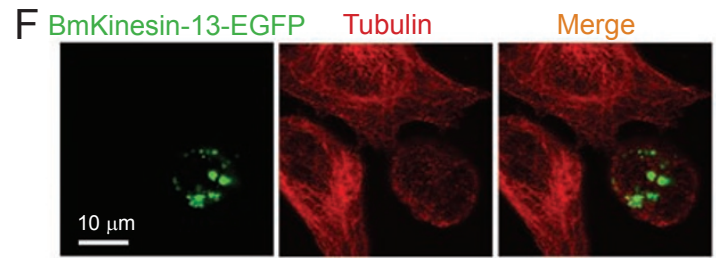

Figure 8 BmKinesin-13 overexpression disrupts microtubule network in vivo. (A) In the control sample only expressing EGFP, the microtubule network was kept intact in $92 \%$ cells. (B) In the majority of cells (89.4\%) overexpressing BmKinesin-13-EGFP, the microtubule network was disrupted. (C) Colocalization of BmKinesin-13 with microtubules. In a minority of infected population $(0.5 \%)$, BmKinesin-13-EGFP colocalized with integrated microtubules. (D) BmKinesin-13 diffused, with microtubules intact. A small portion of $\mathrm{BmN}$ cells $(10.1 \%)$ exhibited the phenotype that BmKinesin-13-EGFP diffused throughout the entire cell, but possessed integrated microtubule network. The BmN cells in $\mathbf{C}$ and $\mathbf{D}, 11 \%$ in total, possessed integrated filamentous microtubules. (E) Qualification of three different patterns mentioned above in BmNPV-BmKinesin-13-EGFP- and BmNPV-EGFP-infected cells. (F) Overexpression of BmKinesin-13 in HeLa cells leads to disruption of microtubule network. silkworm is likely to have a kinesin superfamily as large as Dm.

The expression patterns of the KLPs depend on the cell type as well as developmental stage of the fifth-instar (Figure 3). Their different tissue distribution patterns suggested distinct physiological functions. BmKinesin-6 belongs to Kinesin-6 subfamily, with its homologues DmKLP54E and MmKlp17 functioning in cytokinesis [37]. BmKinesin-7, homologous to DmCana and DmCmeta in fly and to HsCENP-E in humans, belongs to Kinesin-7 subfamily, whose members are reported to capture microtubules at kinetochore during mitosis [3]. Both BmKinesin-6 (Figure 3B) and BmKinesin-7 (Figure $3 \mathrm{C}$ ) are abundant in the fifth-instar day-3 silkworm brain, indicating significant cell proliferation in the developing brain. BmKinesin-14A belongs to Kinesin-14A subfamily and has a motor domain located at the $\mathrm{C}$-terminal region. DmNcd, the homologue of BmKinesin-14A in Dm, is a minus-end-directed microtubule motor and focuses kinetochore microtubule bundles during the formation of metaphase spindle [10]. Recently, Drosophila DmNcd and fission yeast Klp2, both from Kinesin-14 subfamily, are reported to assemble bundles of parallel microtubules, but cause sliding of anti-parallel microtubules $[12,13]$. The extraordinarily high expression level of BmKinesin-14A in silkworm brain (Figure 3E) implies its role in minus-end-directed transport and microtubule organization in neurites. As for silkgland cells, the MSG and PSG cells do not proliferate, but instead become hypertrophic [19]. Once entering the fifth-instar period, these silkgland cells are dramatically enlarged, and begin to synthesize and secrete a substantial amount of fibroin for silk production $[18,20]$. We suspect that MSG and PSG cells may compensate this functional change by reducing the expression levels of proteins unrelated to cell enlargement and intracellular transport, leading to the daily decline of BmKinesin-6, BmKinesin-7, and BmKinesin-14A in the fifth-instar MSG and PSG (Figure $3 \mathrm{~B}, 3 \mathrm{C}$ and $3 \mathrm{E})$.

Bioinformatic analysis showed that the motor domain of BmKinesin-13 was located in the middle as its homologues DmKLP10A in Drosophila and HsMCAK in humans (Figure 1 and Supplementary information, Figure S3). BmKinesin-13, which may possess microtubuledepolymerizing activity (Figure 8), is a member of Kinesin-13 subfamily. In the fifth-instar MSG and PSG, the relative mRNA level of BmKinesin-13 increases during the first five days, and declines on days 6 and 7 (Figure 3D). These observed BmKinesin-13 expression changes might be tightly correlated with its physiological function and PSG developmental stages. PSG exhibits substantial cell enlargement and intensive secretion once it 
enters the fifth-instar period, thus we speculate that BmKinesin-13 accumulates to disassemble/reorganize the microtubule network to facilitate intracellular transport. During the last two days of the fifth-instar, the decrease of BmKinesin-13 in MSG and PSG may be due to the programmed cell death (PCD) caused by pupal metamorphosis [38].

BmKinesin-1 belongs to Kinesin-1 subfamily, and is homologous to DmKHC in Drosophila and KIF5 in mammals. In Drosophila, mutations of both and light chain disrupt axonal transport, causing motor neuron disease phenotypes [39]. In mouse, BmKinesin-1's homologue KIF5 has three members, namely, neuron-specific KIF5A and KIF5C, and ubiquitous KIF5B. KIF5s transport cargoes like various vesicles, organelles, and mRNA complexes [14, 40, 41]. In the fifth-instar PSG of silkworm, BmKinesin-1 expression increases and peaks at day 5 (Figures $3 \mathrm{~A}$ and $4 \mathrm{C}$ ), the time of substantial synthesis and secretion of fibroin [42]. As fibroin synthesis slows down, both BmKinesin-1 and tubulin decrease at the 6 day (Figure 4C), possibly due to the PCD [38].

In PSG cells, when fibroin is synthesized substantially, the mRNA transcripts and fibroin-containing vesicles accumulate significantly. The fibroin-containing vesicles are transported from basal part of the polarized PSG cells to the apical cytoplasm along the radial microtubule system, and secrete fibroin into the glandular lumen when reaching the luminal surface [23, 24]. Our study has suggested that BmKinesin-1, as a potential motor protein, transports fibroin-containing vesicles and exuperantiaassociated RNP complexes along microtubules. However, the mechanisms underlying these interactions remain unknown. Fibroin resides inside the lumen and vesicles, while BmKinesin-1 is a cytosolic protein. Therefore, we could not exclude the possibility that the FLch might interact indirectly with BmKinesin-1-CBD. Further experiments are needed. As for the mRNA transport, motor proteins, including both kinesin and dynein motors, have been demonstrated to participate in RNP complex transport in many eukaryotic cell types $[14,43]$. In Drosophila, DmKHC is required for posterior localization of oskar mRNA [44]. However, in Drosophila oocytes, dynein, but not kinesin, has been reported to actively transport exuperantia, the core component of RNP complex involved in localizing mRNAs [45, 46], to ensure proper localization of the RNP complex [45-47]. Interestingly, our study showed that exuperantia interacted with BmKinesin-1, a Kinesin-1 member, suggesting that in PSG cells, the exuperantia-associated RNP complex could be transported by KLP BmKinesin-1. Further investigations are required to elucidate the molecular mechanism of BmKinesin-1-mediated exuperantia RNP transport, and to explore whether this RNP transport mechanism also exists in Drosophila and other organisms.

It has been previously reported that Kinesin-1 proteins interact with their cargoes either by the kinesin light chain (KLC)-binding domain in the coiled-coil region [48] or by their CBDs [14]. In our GST-BmKinesin-1CBD pull-down experiments, we did not identify silkworm KLC. However, silkworm KLC in PSG extracts could be coimmunoprecipitated with anti-BmKinesin-1 antibody (data not shown), suggesting that KLC exists in silkworm PSG. Future studies are required to reveal the transport mechanisms of BmKinesin-1 and other silkworm kinesins.

Taken together, we propose that silkworm PSG serves as a novel model in the study of kinesin-driven intracellular transport in secretory tissues, and as an important supplement to the previously well-characterized model systems, such as neurons [14, 15], flagellated cells [16], and pigment cells [17]. By using this model, many novel transporting and secreting pathways may be identified, leading to a better understanding of secretion physiology and related human diseases.

\section{Materials and Methods}

\section{Bombyx mori strain and cell culture}

The $B$. mori strain (p50) was used in all experiments. The embryos were incubated in a humidified chamber at room temperature $\left(\sim 25^{\circ} \mathrm{C}\right)$. After hatching, the larvae were reared on the artificial diet offered by the Chinese Academy of Agricultural Sciences. $\mathrm{BmN}$ cells were maintained at $27{ }^{\circ} \mathrm{C}$ in $\mathrm{TC}-100$ insect medium (Sigma Aldrich), supplemented with 10\% fetal bovine serum (FBS) (GIBCO BRL). HeLa cells were cultured in Dulbecco's modified Eagle's medium (GIBCO BRL) supplemented with 10\% FBS at $37^{\circ} \mathrm{C}$ with $5 \% \mathrm{CO}_{2}$.

\section{Bioinformatic analysis}

Sequences for cloning analysis were mainly obtained from http://silkworm.genomics.org.cn/ [21, 49] and http://papilio. ab.a.u-tokyo.ac.jp/silkbase/ [22]. The motor domains and coiledcoil regions of kinesins were predicted respectively by SMART at http://smart.embl-heidelberg.de/ $[50,51]$ and COILS at http:// www.ch.embnet.org/software/COILS form.html [52]. The amino acid sequences of motor domains of $\sim 150$ kinesin superfamily members and the five silkworm kinesins were aligned using MAFFT software at http://align.bmr.kyushu-u.ac.jp/mafft/software/ [53]. The neighbouring-joining tree was inferred and decorated by MEGA 3.1 [54], and was arbitrarily rooted using ScSmy1 [36]. Most sequences of kinesin motor domains for phylogenic analysis were derived from Kinesin Home Page http://www.proweb.org/kinesin/ [36] and NCBI http:/www.ncbi.nlm.nih.gov/. The standardized kinesin nomenclature, based on 14-family designation, was prevailingly accepted.

\section{RNA isolation and cDNA cloning}

Silkworm larvae were anesthetized on shaved ice for more than 
$10 \mathrm{~min}$, and were dissected under Lumar.V12 stereomicroscope (ZEISS). The dissected PSGs were pulverized in liquid nitrogen and then homogenized in TRIzol reagent (Invitrogen). Total RNA was extracted as described previously (Molecular Cloning, 3rd Edition). To precipitate RNA from the aqueous phase, we used 0.25 volume of isopropanol and 0.25 volume of RNA precipitation solution $(0.8 \mathrm{M}$ disodium citrate and $1.2 \mathrm{M} \mathrm{NaCl})$ instead of 0.5 volume of isopropanol [55]. This modification greatly eliminated the contamination of proteoglycans and polysaccharides, which had been reported to prevent the solubilization of precipitated RNA and to inhibit RT-PCR. Subsequently, $2 \mu \mathrm{g}$ of total RNA and Superscript III Reverse Transcriptase (Invitrogen) were used to synthesize the first-strand cDNA. The synthesized cDNA was stored at $-20^{\circ} \mathrm{C}$ for subsequent kinesin cloning and real-time PCR.

Five silkworm kinesins, namely, BmKinesin-1, BmKinesin-6, BmKinesin-7, BmKinesin-13, and BmKinesin-14A, were amplified $\left(30\right.$ cycles of $94^{\circ} \mathrm{C}$ for $30 \mathrm{~s}$ and $60{ }^{\circ} \mathrm{C}$ for $30 \mathrm{~s}$ and $72{ }^{\circ} \mathrm{C}$ for 3 min) using LA Taq DNA polymerase (Takara). A single band of predicted size was observed in each sample by electrophoresis. Then, the PCR products were cloned into BamHI/StuI site on pFastBac-Dual (Invitrogen) or BglII/EcoRI site on pEGFP-N2 vector (CLONTECH Laboratories). The constructed clones were delivered for sequencing (Invitrogen). To rule out errors introduced by PCR, we delivered at least three clones of each band for sequencing (primers: F-BmKinesin-1-GGG GGATCC ATG GCA GCC GAT CGC GAG ATTG and R-BmKinesin-1-GGG AGGCCT ACT TTC ATC TCT AGC GCC GACG; F-BmKinesin-6GGG GGATCC ATG ATG GAT CAA CGT TAT TCG GAC and R-BmKinesin-6-GGG AGGCCT CTG TAT TAG TTC GAA GTG CAG TCT; F-BmKinesin-7-GGG GGATCC ATG AGT GAT AAT ATC AAA GTG GTT GT and R-BmKinesin-7-GGG $\underline{\text { AGGCCT }}$ GTA ATA CAT ACC GAG CTC GAT GAT; F-BmKinesin-13GGG GGATCC ATG CGG GTG TCG AAT GTG GGC and RBmKinesin-13-GGG AGGCCT GTT GTG TAT GCG GCG CGA GATC; F-BmKinesin-14A-GGG GGATCC ATG TCC AAG ATA CCA AAA CTA CCAA and R-BmKinesin-14A-GGG $\underline{\text { AGGCCT }}$ AGT GTC GAC CAT ACA TAG GTT CTT).

\section{Real-time quantitative PCR}

Reverse transcription products of different tissues at different times were amplified by ABI 7300 Detection System (Applied Biosystems) using the SYBR Green PCR Master Mix (Applied Biosystems) according to the manufacturer's instructions. The relative expression levels of each of the five kinesins were normalized to ribosomal protein L3 (RpL3), which has been shown to have the most ubiquitous expression in silkworm [56]. $2^{-\Delta \Delta C}$ method was used to calculate relative expression changes [57]. With the help of dissociation curve analysis and the sequencing of PCR products, pairs of specific primers of each cDNAs were designed and selected, without any primer dimers or unspecific amplification detected (primers: F-RT-BmKinesin-1-TGG TGA ATG TCG CCT TTT GAT and R-RT-BmKinesin-1-ATC GAC TCT GCT AGC GAA GCA; F-RT-BmKinesin-6-TGC AGT GGG TCA AAA GAT TGC and R-RT-BmKinesin-6-CAT ACG ACG GTA CTT GTC ACG AA; F-RT-BmKinesin-7-ATC AGC ACC CGT CGC AAA and R-RT-BmKinesin-7-CGA GTG CAA AGC CGA TAT TG; F-RT-BmKinesin-13-GAT CAG CGT CCC CAC CAA and R-RT-BmKinesin-13-AAT ACT TCG TGA GGT CCA CTT TGT T; F-RT-BmKinesin-14A-AAA TGG CCC ACC TGT TTC
TG and R-RT-BmKinesin-14A-TCG GAA TTG TTG TCG ATG ATG; F-RT-BmRpL3-GAG GTC CCG TCG TCA TCG T and RRT-BmRpL3-CAG GTT TGC TAG GGT CGT CTT T).

\section{Antibody production}

BmKinesin-1 antibody production The regions corresponding to the motor domain (amino acid 10-341) (primers: F-BmKinesin-1-motor domain-GGG GGATCC GAA GAC AGT ATC AGA GTG GTA TGC and R-BmKinesin-1-motor domain-GGG GTC GAC TCA CTC ATT GAC ACA TAC AAC ATT CT TGA) and the tail domain (amino acid 746-964) (primers: F-BmKinesin1-tail domain- GGG GAATTC CTC CAG GAC GAC TAC GAG AAGC and R-BmKinesin-1-tail domain- GGG GTCGAC TCA ACT TTC ATC TCT AGC GCC GAC) were amplified by PCR. The motor domain was cloned into BamHI/SalI and the tail domain intoEcoRI/SalI in the expression vector pGEX-6P-1. GSTBmKinesin-1 fusion proteins were expressed in E. coli BL21. Cells were collected and all the following steps were performed at $4{ }^{\circ} \mathrm{C}$. The collected cells were resuspended in the lysis buffer $(20$ $\mathrm{mM}$ Tris, $120 \mathrm{mM} \mathrm{NaCl}, 5 \mathrm{mM}$ EDTA (pH 7.5)) and then lysed by FRENCH Press (Thermo). The lysate was subjected to centrifugation at $130000 \times g$ for $30 \mathrm{~min}$, and the resulting supernatant was harvested and incubated with Glutathione-Sepharose 4B beads (Amersham) for $2 \mathrm{~h}$. The fusion proteins were purified and eluted in the recommended buffer (50 mM glutathione ( $\mathrm{pH} 7.5), 0.5 \mathrm{M}$ $\mathrm{NaCl}, 0.1 \%$ TritonX-100, $1 \mathrm{mM}$ DTT) according to the manufacturer's protocol. The purified GST fusion proteins were utilized as antigens to immunize both rabbits and mice.

BmKinesin-13 antibody production One fragment (amino acid 400-623) was amplified by RT-PCR (primers: F-BmKinesin13-fragment-TTT GAATTC ATC GAC CTG GCG GGC AAC GA and R-BmKinesin-13-fragment-TTT CTCGAG GTT GTG TAT GCG GCG CGA GAT). The GST-BmKinesin13 fusion protein was produced as described for the GST-BmKinesin1, and was then used to immunize mice.

FLch antibody production Both the full-length FLch protein and the truncated $\mathrm{N}$-/C-terminal recombinant proteins were predicted to be insoluble. We amplified FLch from silkworm PSG cDNA library as previously reported [35], and subcloned into the BamHI/XhoI site on vector pET-28a (+) (Novagen) (primers: FFLch-GGG GGATCC ATG AAG CCT ATA TTT TTG GTA TTA CTC and R-FLch-GGG CTCGAG GAC GTG AAC CTG GCT GGC TGC). This plasmid was transfected into E. coli to express His-tagged fusion protein. His-tagged proteins were expressed, and purified under denaturing conditions as described in ProBond ${ }^{\mathrm{TM}}$ Purification System (Invitrogen). We immunized rabbits with the dialyzed protein.

\section{Transfection and immunofluorescence}

HeLa cells were transiently transfected by calcium phosphate precipitation at $\sim 60 \%$ confluency. At $36-48 \mathrm{~h}$ post-transfection, cells were subjected to immunofluorescence microscopy. For immunofluorescence, the cells were washed in PBS and fixed with freshly prepared $4 \%$ paraformaldehyde in PBS for $15 \mathrm{~min}$ at 37 ${ }^{\circ} \mathrm{C}$. The samples were then permeabilized with $0.1 \%$ Triton X-100 in PBS for 15 min. After blocking with 3\% BSA in PBS for 30 min, cells were incubated with primary antibodies overnight at 
$4{ }^{\circ} \mathrm{C}$. We used Alexa Fluor 488- and/or 568- conjugated goat anti-mouse $\mathrm{IgG}$ and/or anti-rabbit $\mathrm{IgG}$ as secondary antibodies (Molecular Probes). Samples were observed with IX71 inverted fluorescence microscope (OLYMPUS) or the TCS SP2 confocal microscope (Leica) equipped with a $\times 100 / 1.4$ numerical aperture oil immersion objective lens.

For immunofluorescence of cryosections, the whole silkworm body of the third-instar larvae were fixed with 3\% paraformaldehyde in PBS at room temperature. The samples are sectioned with a Leica cryostat CM1850 following the manufacturer's instructions. The sections were treated with methanol at $-20{ }^{\circ} \mathrm{C}$, and were processed with immunofluorescence using primary antibodies (rabbit anti-FLch polyclonal antibody and mouse anti-BmKinesin-1 polyclonal antibody) and secondary antibodies mentioned above. Samples were observed with TCS SP2 confocal microscope (Leica).

\section{Recombinant baculovirus and infection}

Five kinesin cDNAs were subcloned into the BamHI/StuI site on pFastBac-Dual vector (Invitrogen). We further inserted the EGFP/EYFP into the StuI/XbaI site to construct pFastBac-DualBmKLP-EGFP/EYFP to express BmKLP-EGFP/EYFP fusion proteins under the polyhedron promoter. The recombinant BmNPV baculoviruses were generated according to the manufacturer's protocol (Invitrogen) and were used to infect BmN cells and silkworm larvae. BmN cells were fixed $48 \mathrm{~h}$ post-infection and subjected to immunofluorescence microscopy. The statistical analysis was based on at least 500-cell counting. The recombinant BmNPV baculoviruses, encoding BmKinesin-14A-EYFP or BmKinesin-1EGFP fusion protein, were injected into the hemocoel of silkworm larvae newly ecdysed into fourth-instar. The PSGs were dissected more than 7 days after injection, immersed in TC-100 medium (Sigma), and then directly subjected to microscopy observation. The samples were photographed with inverted TCS SP2 confocal microscopy (Leica).

\section{Pull-down analysis and protein identification}

The nucleotide sequence of BmKinesin-1-CBD (amino acids 827-964) was inserted into pGEX-6P-1 (primers: F-BmKinesin-1CBD-GGG GAATTC ACC CTT GCA CAA AAA CAA AAG ATC TC and R-BmKinesin-1-CBD-GGG GTCGAC TCA ACT TTC ATC TCT AGC GCC GAC). The fusion protein GST-BmKinesin1-CBD was expressed by standard E. coli expression system. We incubated the fusion protein with Glutathione-Sepharose $4 \mathrm{~B}$ beads (Amersham) to make affinity column for pull-down study. We homogenized PSGs of more than 500 heads of the fifth-instar silkworm larvae in lysis buffer (20 mM HEPES ( $\mathrm{pH} 7.4), 150$ $\mathrm{mM} \mathrm{NaCl}, 1 \mathrm{mM}$ EDTA, $0.5 \%$ Nonidet P-40) containing protease inhibitor cocktail (Roche). The centrifugated homogenate was incubated overnight with the affinity column. After wash with lysis buffer, we eluted the column with high-salt buffers with stepwise increases in $\mathrm{NaCl}$ concentration $(200 \mathrm{mM}, 400 \mathrm{mM}, 600 \mathrm{mM}$, and $800 \mathrm{mM}$ ), and harvested sequential fractions from GST- and GSTBmKinesin-1-CBD pull-down columns. To identify the proteins separated on SDS-PAGE gels, we excised and digested the bands with sequence-grade modified trypsin (Promega). The prepared samples were subjected to a LCQ Deca XP Plus Analyzer liquid chromatography/mass spectrometry (Finnigan).

\section{Immunoblotting analysis}

Proteins were separated by SDS-PAGE and transferred onto PVDF membranes (Millipore) in a semidry transfer cell (BIORAD). After blocking, the membranes were probed with primary antibodies at $4{ }^{\circ} \mathrm{C}$ overnight. Alkaline phosphatase-conjugated goat anti-mouse and goat anti-rabbit IgG antibodies (Jackson ImmunoResearch Laboratories) were used as secondary antibodies. The results were revealed by the NBT-BCIP (Promega) reaction.

\section{Whole-mount in situ hybridization}

For whole mount in situ hybridization experiment, brains of silkworm larvae were isolated and dehydrated by methanol after fixation. Prehybridization solution was prepared as reported previously [58]. After $2 \mathrm{~h}$ of prehybridization at $50{ }^{\circ} \mathrm{C}$, DIG-labelled RNA probe, produced by in vitro transcription system (Promega) and DIG RNA Mix (Roche), were added into the same buffer. Probe-target hybrids were localized with Anti-DIG-AP (Roche), and visualized by NBT/BCIP (Promega) according to the manufacturer's instructions.

\section{Acknowledgments}

We wish to thank Prof GZ Zhang and Prof ZF Zhang at the Sericultural Research Institute of the Chinese Academy of Agricultural Sciences for B. mori strain and silkworm artificial diet, respectively. This work was supported by the National Natural Science Foundation of China (30670659, 30771086, 30721064), the Major State Basic Research Development Program of China (973 Program) (2006CB500700, 2006CB910700, 2010CB833705), and the National High Technology Research and Development Program of China (863 Program) (2006AA10A119).

\section{References}

1 Vale RD, Reese TS, Sheetz MP. Identification of a novel force-generating protein, kinesin, involved in microtubulebased motility. Cell 1985; 42:39-50.

2 Lawrence CJ, Dawe RK, Christie KR et al. A standardized kinesin nomenclature. J Cell Biol 2004; 167:19-22.

3 Miki H, Okada Y, Hirokawa N. Analysis of the kinesin superfamily: insights into structure and function. Trends Cell Biol 2005; 15:467-476.

4 Yildiz A, Tomishige M, Gennerich A, Vale RD. Intramolecular strain coordinates kinesin stepping behavior along microtubules. Cell 2008; 134:1030-1041.

5 Alonso MC, Drummond DR, Kain S, Hoeng J, Amos L, Cross RA. An ATP gate controls tubulin binding by the tethered head of kinesin-1. Science 2007; 316:120-123.

6 Teng J, Rai T, Tanaka Y, et al. The KIF3 motor transports Ncadherin and organizes the developing neuroepithelium. Nat Cell Biol 2005; 7:474-482.

7 Hunter AW, Caplow M, Coy DL, et al. The kinesin-related protein MCAK is a microtubule depolymerase that forms an ATP-hydrolyzing complex at microtubule ends. Mol Cell 2003; 11:445-457.

8 Rogers GC, Rogers SL, Schwimmer TA, et al. Two mitotic kinesins cooperate to drive sister chromatid separation during anaphase. Nature 2004; 427:364-370. 
9 Tan D, Asenjo AB, Mennella V, Sharp DJ, Sosa H. Kinesin13 s form rings around microtubules. J Cell Biol 2006; 175:2531 .

10 Goshima G, Nedelec F, Vale RD. Mechanisms for focusing mitotic spindle poles by minus end-directed motor proteins. $J$ Cell Biol 2005; 171:229-240.

11 Furuta K, Toyoshima YY. Minus-end-directed motor Ned exhibits processive movement that is enhanced by microtubule bundling in vitro. Curr Biol 2008; 18:152-157.

12 Fink G, Hajdo L, Skowronek KJ, Reuther C, Kasprzak AA, Diez S. The mitotic kinesin-14 Ned drives directional microtubule-microtubule sliding. Nat Cell Biol 2009; 11:717-723.

13 Braun M, Drummond DR, Cross RA, McAinsh AD. The kinesin-14 Klp2 organizes microtubules into parallel bundles by an ATP-dependent sorting mechanism. Nat Cell Biol 2009; 11:724-730.

14 Hirokawa N, Takemura R. Molecular motors and mechanisms of directional transport in neurons. Nat Rev Neurosci 2005; 6:201-214.

15 Goldstein AY, Wang X, Schwarz TL. Axonal transport and the delivery of pre-synaptic components. Curr Opin Neurobiol 2008; 18:495-503.

16 Rosenbaum JL, Witman GB. Intraflagellar transport. Nat Rev Mol Cell Biol 2002; 3:813-825.

17 Kural C, Serpinskaya AS, Chou YH, Goldman RD, Gelfand VI, Selvin PR. Tracking melanosomes inside a cell to study molecular motors and their interaction. Proc Natl Acad Sci USA 2007; 104:5378-5382.

18 Wurm FM. Human therapeutic proteins from silkworms. Nat Biotechnol 2003; 21:34-35.

19 Tashiro Y, Morimoto T, Matsuura S, Nagata S. Studies on the posterior silk gland of the silkworm, Bombyx mori. I. Growth of posterior silk gland cells and biosynthesis of fibroin during the fifth larval instar. J Cell Biol 1968; 38:574-588.

20 Inoue S, Tanaka K, Arisaka F, Kimura S, Ohtomo K, Mizuno $\mathrm{S}$. Silk fibroin of Bombyx mori is secreted, assembling a high molecular mass elementary unit consisting of H-chain, Lchain, and P25, with a 6:6:1 molar ratio. J Biol Chem 2000; 275:40517-40528.

21 Xia Q, Zhou Z, Lu C, et al. A draft sequence for the genome of the domesticated silkworm (Bombyx mori). Science 2004; 306:1937-1940.

22 Mita K, Morimyo M, Okano K, et al. The construction of an EST database for Bombyx mori and its application. Proc Natl Acad Sci USA 2003; 100:14121-14126.

23 Sasaki S, Tashiro Y. Studies on the posterior silk gland of the silkworm Bombyx mori. VI. Distribution of microtubules in the posterior silk gland cells. J Cell Biol 1976; 71:565-574.

24 Sasaki S, Nakajima E, Fujii-Kuriyama Y, Tashiro Y. Intracellular transport and secretion of fibroin in the posterior silk gland of the silkworm Bombyx mori. J Cell Sci 1981; 50:1944.

25 Ohnishi A, Hull JJ, Matsumoto S. Targeted disruption of genes in the Bombyx mori sex pheromone biosynthetic pathway. Proc Natl Acad Sci USA 2006; 103:4398-4403.

26 Miao Y, Zhang Y, Nakagaki K, et al. Expression of spider flagelliform silk protein in Bombyx mori cell line by a novel Bac-to-Bac/BmNPV baculovirus expression system. Appl Microbiol Biotechnol 2006; 71:192-199.
27 Tomita M, Munetsuna H, Sato T, et al. Transgenic silkworms produce recombinant human type III procollagen in cocoons. Nat Biotechnol 2003; 21:52-56.

28 Chen W, Foss M, Tseng KF, Zhang D. Redundant mechanisms recruit actin into the contractile ring in silkworm spermatocytes. PLoS Biol 2008; 6:e209.

29 Okano K, Takada N, Kobayashi M, Maekawa H. cDNA structure and characterization of a kinesin-like protein from the silkworm Bombyx mori. Insect Mol Biol 1994; 3:195-200.

30 Stewart RJ, Pesavento PA, Woerpel DN, Goldstein LS. Identification and partial characterization of six members of the kinesin superfamily in Drosophila. Proc Natl Acad Sci USA 1991; 88:8470-8474.

31 Lippincott-Schwartz J, Cole NB, Marotta A, Conrad PA, Bloom GS. Kinesin is the motor for microtubule-mediated Golgi-to-ER membrane traffic. J Cell Biol 1995; 128:293306.

32 Helenius J, Brouhard G, Kalaidzidis Y, Diez S, Howard J. The depolymerizing kinesin MCAK uses lattice diffusion to rapidly target microtubule ends. Nature 2006; 441:115-119.

33 Manning AL, Ganem NJ, Bakhoum SF, Wagenbach M, Wordeman L, Compton DA. The kinesin-13 proteins Kif2a, $\mathrm{Kif} 2 \mathrm{~b}$, and Kif2c/MCAK have distinct roles during mitosis in human cells. Mol Biol Cell 2007; 18:2970-2979.

34 Nakagawa T, Tanaka Y, Matsuoka E, et al. Identification and classification of 16 new kinesin superfamily (KIF) proteins in mouse genome. Proc Natl Acad Sci USA 1997; 94:9654-9659.

35 Kikuchi Y, Mori K, Suzuki S, Yamaguchi K, Mizuno S. Structure of the Bombyx mori fibroin light-chain-encoding gene: upstream sequence elements common to the light and heavy chain. Gene 1992; 110:151-158.

36 Dagenbach EM, Endow SA. A new kinesin tree. J Cell Sci 2004; 117:3-7.

37 Hill E, Clarke M, Barr FA. The Rab6-binding kinesin, Rab6KIFL, is required for cytokinesis. EMBO J 2000; 19:57115719.

38 Kakei M, Iwami M, Sakurai S. Death commitment in the anterior silk gland of the silkworm, Bombyx mori. J Insect Physiol 2005; 51:17-25.

39 Gindhart JG, Jr, Desai CJ, Beushausen S, Zinn K, Goldstein LS. Kinesin light chains are essential for axonal transport in Drosophila. J Cell Biol 1998; 141:443-454.

$40 \mathrm{Li}$ JY, Wu CF. Perspectives on the origin of microfilaments, microtubules, the relevant chaperonin system and cytoskeletal motors - a commentary on the spirochaete origin of flagella. Cell Res 2003; 13:219-227.

41 Ni CZ, Wang HQ, Xu T, Qu Z, Liu GQ. AtKP1, a kinesinlike protein, mainly localizes to mitochondria in Arabidopsis thaliana. Cell Res 2005; 15:725-733.

42 Suzuki Y, Suzuki E. Quantitative measurements of fibroin messenger RNA synthesis in the posterior silk gland of normal and mutant Bombyx mori. J Mol Biol 1974; 88:393-407.

43 Kindler S, Wang H, Richter D, Tiedge H. RNA transport and local control of translation. Annu Rev Cell Dev Biol 2005; 21:223-245.

44 Brendza RP, Serbus LR, Duffy JB, Saxton WM. A function for kinesin I in the posterior transport of oskar mRNA and Staufen protein. Science 2000; 289:2120-2122.

45 Wilhelm JE, Mansfield J, Hom-Booher N, et al. Isolation of a 
ribonucleoprotein complex involved in mRNA localization in Drosophila oocytes. J Cell Biol 2000; 148:427-440.

46 Mische S, Li M, Serr M, Hays TS. Direct observation of regulated ribonucleoprotein transport across the nurse cell/oocyte boundary. Mol Biol Cell 2007; 18:2254-2263.

47 Riechmann V, Ephrussi A. Par-1 regulates bicoid mRNA localisation by phosphorylating Exuperantia. Development 2004; 131:5897-5907.

48 Wozniak MJ, Allan VJ. Cargo selection by specific kinesin light chain 1 isoforms. EMBO J 2006; 25:5457-5468.

49 Wang J, Xia Q, He X, et al. SilkDB: a knowledgebase for silkworm biology and genomics. Nucleic Acids Res 2005; 33:D399-D402.

50 Schultz J, Milpetz F, Bork P, Ponting CP. SMART, a simple modular architecture research tool: identification of signaling domains. Proc Natl Acad Sci USA 1998; 95:5857-5864.

51 Letunic I, Copley RR, Pils B, Pinkert S, Schultz J, Bork P. SMART 5: domains in the context of genomes and networks. Nucleic Acids Res 2006; 34:D257-D260.

52 Lupas A, Van Dyke M, Stock J. Predicting coiled coils from protein sequences. Science 1991; 252:1162-1164.
53 Katoh K, Misawa K, Kuma K, Miyata T. MAFFT: a novel method for rapid multiple sequence alignment based on fast Fourier transform. Nucleic Acids Res 2002; 30:3059-3066.

54 Kumar S, Tamura K, Nei M. MEGA3: integrated software for molecular evolutionary genetics analysis and sequence alignment. Brief Bioinform 2004; 5:150-163.

55 Chomczynski P, Mackey K. Short technical reports. Modification of the TRI reagent procedure for isolation of RNA from polysaccharide- and proteoglycan-rich sources. Biotechniques 1995; 19:942-945.

56 Matsuoka T, Fujiwara H. Expression of ecdysteroid-regulated genes is reduced specifically in the wing discs of the wingdeficient mutant (fl) of Bombyx mori. Dev Genes Evol 2000; 210:120-128.

57 Livak KJ, Schmittgen TD. Analysis of relative gene expression data using real-time quantitative PCR and the 2(-Delta Delta C(T)) Method. Methods 2001; 25:402-408.

58 Yamanaka N, Hua YJ, Mizoguchi A, et al. Identification of a novel prothoracicostatic hormone and its receptor in the silkworm Bombyx mori. J Biol Chem 2005; 280:14684-14690.

(Supplementary information is linked to the online version of the paper on the Cell Research website.) 